\title{
A Review of Artificial Lateral Line in Sensor Fabrication and Bionic Applications for Robot Fish
}

\author{
Guijie Liu, ${ }^{1,2}$ Anyi Wang, ${ }^{1,2}$ Xinbao Wang, ${ }^{1,2}$ and Peng Liu ${ }^{1,2}$ \\ ${ }^{1}$ Department of Engineering, Ocean University of China, Qingdao, Shandong 266071, China \\ ${ }^{2}$ Key Laboratory of Ocean Engineering of Shandong Province, Ocean University of China, Qingdao, Shandong 266071, China \\ Correspondence should be addressed to Guijie Liu; paperlgj@163.com
}

Received 15 June 2016; Revised 17 August 2016; Accepted 25 October 2016

Academic Editor: Jean Slawinski

Copyright (c) 2016 Guijie Liu et al. This is an open access article distributed under the Creative Commons Attribution License, which permits unrestricted use, distribution, and reproduction in any medium, provided the original work is properly cited.

\begin{abstract}
Lateral line is a system of sense organs that can aid fishes to maneuver in a dark environment. Artificial lateral line (ALL) imitates the structure of lateral line in fishes and provides invaluable means for underwater-sensing technology and robot fish control. This paper reviews ALL, including sensor fabrication and applications to robot fish. The biophysics of lateral line are first introduced to enhance the understanding of lateral line structure and function. The design and fabrication of an ALL sensor on the basis of various sensing principles are then presented. ALL systems are collections of sensors that include carrier and control circuit. Their structure and hydrodynamic detection are reviewed. Finally, further research trends and existing problems of ALL are discussed.
\end{abstract}

\section{Introduction}

The lateral line is a collection of sense organs that enables fishes to maneuver in a dark environment [1]. Fishes have two types of lateral line receptors, namely, electrosensory and mechanosensory. The mechanosensory lateral line system exists in all fishes and aquatic amphibians and senses water movements around the skin surface [2]. Artificial lateral line (ALL) is a bionic device that mainly imitates mechanosensory lateral line; it can detect underwater hydrodynamic stimuli and assist in navigating autonomous underwater vehicles (AUVs) and robot fishes. With the development of modern manufacturing technology, many scholars worldwide have studied ALL. This paper provides an overview of ALL, including ALL sensors and systems. ALL system is a special carrier comprising ALL sensors and control circuit. ALL sensor provides a novel approach to underwater flow sensing, whereas ALL systems help to improve the maneuverability of underwater vehicles and robot fishes.

ALL sensors can be classified into two types according to location. One stands on the carrier surface, and the other is located in the canal. Many flow field-measuring technologies exist, such as wheel flow meters, pressure probes, hot-wire anemometers (HWAs), and acoustic Doppler velocimetry.
Compared with these technologies, ALL sensors are flexible and suitable for forming distributed arrays [3]. ALL systems can be categorized into two kinds according to sensor type. One is a collection of off-the-shelf pressure sensors that are protected against humidity and water, such as MS5407AM [4] and SM5420C-030-A-P-S [5]; the other comprises sensing platforms with artificial sensors distributed across the surface.

In the following sections, this paper introduces the biology of fish lateral line and reviews ALL sensors on the basis of sensing modalities, carrier structure, and hydrodynamic detection achievement. Finally, the research tendency and existing problems of ALL are discussed.

\section{Fish Lateral Line}

Stenon [37] first described the lateral line in the 17th century. Hofer [1] found that the lateral line was a hydrodynamic receptor system. Over the last decades, lateral line research has substantially progressed, especially in the fields of biomechanics, neuroanatomy, and biomimetics [3].

The sensing unit of the biological lateral line is the neuromast, a receptor organ that consists of sensory hair cells and support cells covered by a gelatinous cupula [38]. Two 
(a)



FIGURE 1: Lateral line and neuromast of a fish. Black dots represent locations of SNs, and white dots show the approximate locations of canal pores $[6,7]$.

antagonistically oriented hair cell populations are found in each neuromast to encode the direction and displacement [39]. Superficial neuromasts (SNs) are free-standing on the skin or on pedestals grown above the skin, typically aligned in rows on the body of fishes [40] (Figure 1(a)). Canal neuromasts (CNs) are enclosed in fluid-filled canals just below the skin surface. The canal breaks through to the skin and water interface at periodic intervals [6]. Usually, there is one CN between two contiguous pores (Figure 1(b)). Every neuromast possesses a gelatinous cupula packaged with hundreds of hair cells and hair-like cilia [3].

Although hair cells are displacement transducers, the displacement of the cupula is relative to viscous drag and consequently to the velocity of water particles; thus, SNs are mainly sensitive to flow velocity. The frequency response of SNs is defined as cupular deflection $v(H)$ at the height of the beam tip divided by free-stream velocity $U_{\infty}$. The sensitivity is defined as [41]

$$
\begin{aligned}
S_{\mathrm{SN}}(f)= & \frac{v(H)}{U_{\infty}} \\
= & -\frac{i b_{w}}{2 \pi f b_{m}}\left[1-{\frac{i \pi f b_{m} \delta^{4}}{2 E I+i \pi f b_{m} \delta^{4}}}^{(-H(1+i) / \delta)}\right] \\
& +\sum_{j=0}^{3} C_{j}^{\left({ }^{j} H^{4} \sqrt{2 \pi f i b_{m} / E I}\right)} .
\end{aligned}
$$

CNs are regarded as acceleration sensors because the canal fluid velocity is practically proportional to the first full derivative of the flow speed outside the canal [42]. The frequency-dependent sensitivity of CN cupula can be defined as [43]

$$
\begin{aligned}
& S_{\mathrm{CN}}(f)=\frac{Y_{0}(f)}{V_{0}(f)}=\frac{1}{2 \pi f_{t}} \\
& \cdot \frac{1+(\sqrt{2} / 2)(1+i)\left(f / f_{t}\right)^{1 / 2}+(1 / 3) i\left(f / f_{t}\right)}{N_{r}+i\left(f / f_{t}\right)-(\sqrt{2} / 2)(1-i)\left(f / f_{t}\right)^{3 / 2}-(1 / 3)\left(f / f_{t}\right)^{2}}
\end{aligned}
$$

where $f_{t}$ is defined as the transition frequency at which viscous $\left(f<f_{t}\right)$ or inertial $\left(f>f_{t}\right)$ fluid forces dominate the fluid forces acting on the cupula; it can be expressed as

$$
f_{t}=\frac{\mu}{2 \pi \rho_{w} a^{2}},
$$

where $\mu$ is the dynamic viscosity of fluid. $N_{r}$ is defined as the resonance factor that governs the resonance properties of a cupula; it can be expressed as

$$
N_{r}=\frac{K a \rho_{w}}{6 \pi \mu^{2}},
$$

where $\rho_{w}$ is the water density. CNs can also be regarded as pressure-gradient sensors because water acceleration is 


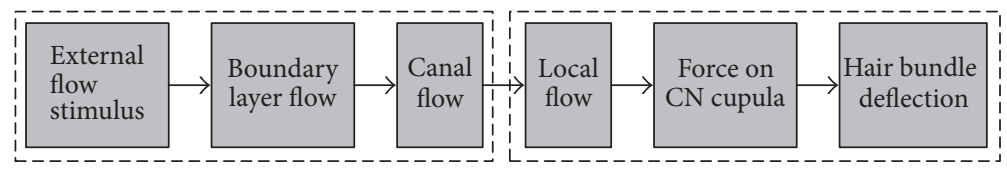

(a)

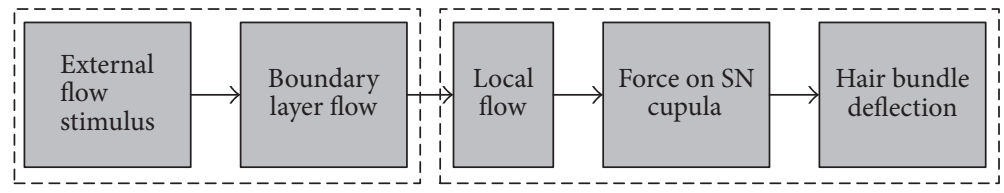

(b)

Figure 2: Propagation path of lateral line neuromasts. (a) Propagation path of CNs. (b) Propagation path of SNs [3].

proportional to the pressure gradients causing the acceleration. Thus, lateral line enables fishes to detect the pressure distributions in their lateral line canals $(\mathrm{CNs})$ as well as the flow velocity and orientation across their body surface (SNs) [43]. SNs can distinguish fields in spatially uniform flow and turbulent flow, while CNs respond only to nonuniform flow fields, such as water fluctuations generated by a vibrating sphere or a swimming fish [44].

Different fish species have various organizations of the peripheral lateral line. This variability includes the quantity and location of SNs and distribution, compartmentalization, and dimension of the canals as well as the quantity, dimension, and location of canal pores $[40,45]$. In most fish species, canals locate above the eye, below the eye, and on the lower jaw. In some fish, for example, Rhodeus, the trunk canal does not across the whole fish body [7]. ALL systems are distributed with a regular symmetric sensory array, which is exposed to water directly similar to fishes, but their quantity is limited to a dozen or less.

\section{Artificial Lateral Line Sensors}

For the theoretical models, biologists have established mathematical models of lateral line to reveal the mechanisms of the sensing capabilities of neuromasts and how they interact with the fluids. Shizhe [3] summarized the mathematical solution and propagation path (Figure 2) of SNs and CNs with respect to their frequency response. The flow around CNs comes from canal flow, whereas the local flow around SNs comes from the boundary layer flow. For both local spherical excitation and external free-stream flow, the general interactions between cupular response and external stimuli are determined by the boundary layer. The biomechanical model of a $\mathrm{CN}$ is a frictionless plate with a rigid hemisphere sliding on it. The model of SNs comprises two attached beams with different flexural stiffness. The frequency response of different neuromasts is related to the propagation path of external stimulus to the cilia bending.

These mathematical models can assist in understanding the theoretical perceptual mechanisms and serve as an inspiration of ALL sensors. At present, many advanced technologies are beneficial for developing a sensor with high sensitivity and resolution that can match the capability of a real fish. By applying plastic deformation magnetic assembly (PDMA) technology and developing microelectromechanical systems (MEMSs), many scholars have designed and fabricated various ALL sensors.

MEMS is a novel batch fabrication technology to develop electromechanical systems, which is similar to those used in integrated circuit design. They can range in size from micrometers to millimeters, which are suitable for developing micro ALL sensors. They have several advantages, namely, small dimension, the possibility of batch fabrication, and low-cost integration with electronics as well as potentially less energy consumption [46]. PDMA is a technique for 3D assembly of microstructures. It has been widely applied to develop various ALL sensors with high controllability. In PDMA, the vertical part of an ALL sensor obtains a plastic deformation caused by the magnetic force generated in an attached magnetic piece with an external magnetic field. PDMA has advantages of simple structure, good electrical connection, simple material, and easy operation [47].

Existing ALL sensors are based on different sensing principles, such as piezoresistive effect, piezoelectric effect, capacitive principle, optical readout principle, and HWA principle. The following section discusses the research status of ALL sensors with different sensing principles.

3.1. Piezoresistive ALL Sensors. The piezoresistive effect is a change in the electrical resistivity of a semiconductor or metal when strain is applied. It has the advantages of high sensitivity, wide band, high signal-to-noise ratio, simple structure, and light weight. Strain gauges are usually used for measurement. Compared with the piezoelectric effect, the piezoresistive effect causes a change only in electrical resistance, not in the electric potential; therefore, it is commonly used to measure the small movement of objects. Although many groups use piezoresistive principles, the output of the direct current response is poor, and a high-input impedance circuit or a charge amplifier must be utilized to overcome this disadvantage.

In ALL sensors that are based on momentum transfer, strain gauges are usually used for measurements; they are usually placed on the most sensitive position by flow to improve sensitivity of gauge [48]. Power supply is needed to tie the sensor in a Wheatstone bridge in piezoresistive 


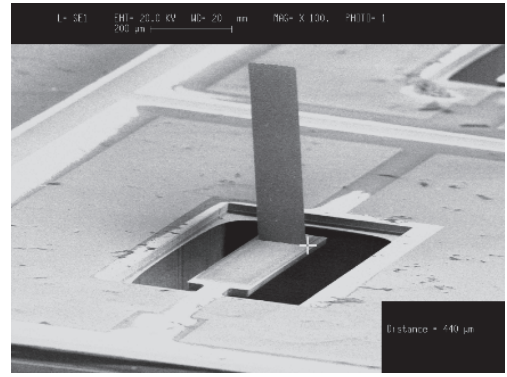

(a)

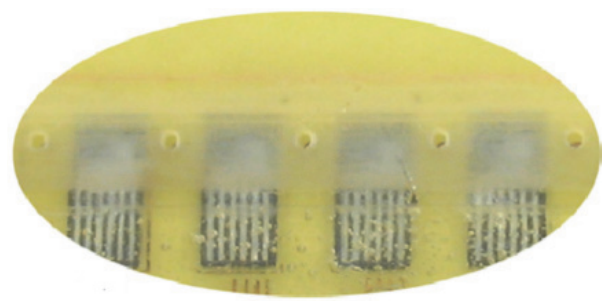

(d)

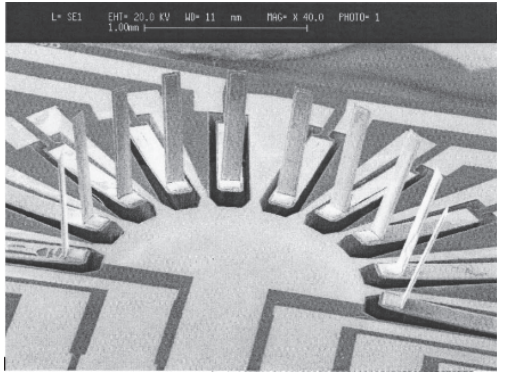

(b)

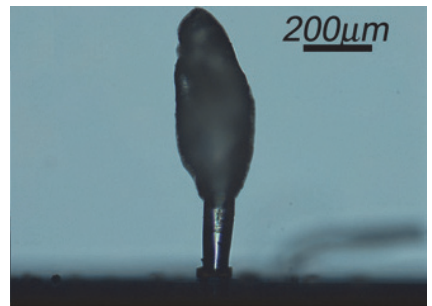

(e)

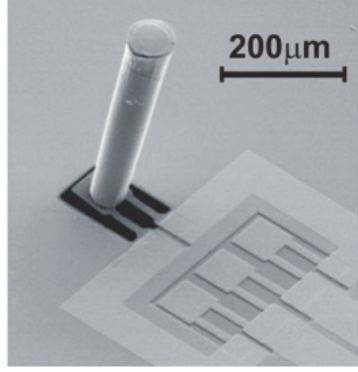

(c)

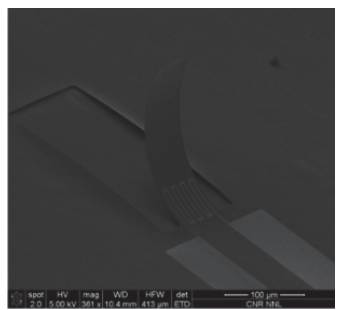

(f)

FIgure 3: Piezoresistive ALL sensors with a vertical hair. (a) First piezoresistive ALL sensor fabricated by Fan et al. [8] in 2002. (b) ALL sensors fabricated by Chen et al. [9] in 2003. (c) New ALL sensor developed by Yang et al. [10] in 2010. (d) ALL canal fabricated in 2011. (e) Optimized ALL sensors developed by McConney et al. [11] in 2009. (f) ALL sensor fabricated with a Nichrome 80/20 alloy [12].

sensors. The voltage output of the Wheatstone bridge, altered by water movements, can be measured [17].

The first piezoresistive ALL sensor was fabricated by Fan et al. [8] in 2002; they provided a typical structure of hair-like piezoresistive ALL sensor (Figure 3(a)). The sensor had three basic parts, namely, a horizontal cantilever, a vertical cilium, and a piezoresistor. The force applied on the vertical cilium generated by local flow caused the bending of a horizontal cantilever beam, which was detected with a stress-strain gauge. The sensor was fabricated by a combination of bulk micromachining technology and an efficient 3D assembly technology. The vertical hair structure was obtained using a PDMA process. The piezoresistor and cantilever beam were realized by doping and etching technologies. Chen et al. [9] compared the above sensor with a hot-wire anemometer and optimized it with respect to high spatial resolution (Figure 3(b)). The vertical structure was rigidly connected to the horizontal substrate, and the strain gauge was placed at the hair base [3].

To improve sensitivity and resolution, Yang et al. [10, 32] developed a new ALL sensor that adopted a high-aspectratio hair structure with a shape of symmetric cylinder. The threshold of the sensor was $0.1 \mathrm{~mm} / \mathrm{s}$, and the sensor survived $55^{\circ}$ deflections (Figure 3(c)). The material of the hair structure was SU-8. In 2011, a new optimization effort was exerted by putting the sensor array in a semicylindrical-shaped canal (diameter $4 \mathrm{~mm}$ ) [33]. The canal structure was fabricated using stereolithography technique. The ALL canal performed well with respect to noise rejection (Figure 3(d)).

To achieve the threshold sensitivities that rival integrated fish flow sensory systems, McConney et al. [11] encapsulated the SU-8 hair structure by dispensing water-soluble polyethylene glycol on it using the precision drop-casting technology. This enhanced the sensitivity by approximately two orders of magnitude (Figure 3(e)). The producing process of artificial hydrogel cupula includes monomer spreading around hair sensor, patterned photopolymerization, and removal of unreacted monomer layer combined with swelling and shaping of dome-shaped cupula [49].

In ALL sensors, the material of the piezoresistor is always based on silicon. Qualtieri et al. [12] utilized a Nichrome $80 / 20$ alloy as a material of piezoresistor and fabricated a stress-driven aluminum nitride (AlN) cantilever ALL sensor, which exploited multilayered cantilever AlN/molybdenum (Mo). Cantilever arrays were fabricated by micromachining techniques including optical lithography and etching processes. This manufacturing approach was promising due to its simple fabrication procedure. The fabricated cantilevers were directional and sensitive and required low forces (Figure 3(f)).

All above ALL sensors have a hair-like structure based on a cantilever beam that is deflected by water movements. The sensor size is in micron level. Other piezoresistive ALL sensors mainly detect underwater pressure fluctuation with a flat structure, and the piezoresistive material is directly mounted onto the substrate to determine the pressure change caused by the fluid flow.

To interpret the hydrodynamic distributions, Fernandez et al. [13] presented experiments demonstrating the discrimination between circular and square cross section obstacles with an array of commercial pressure sensors, and a high-density array of pressure sensors was introduced 


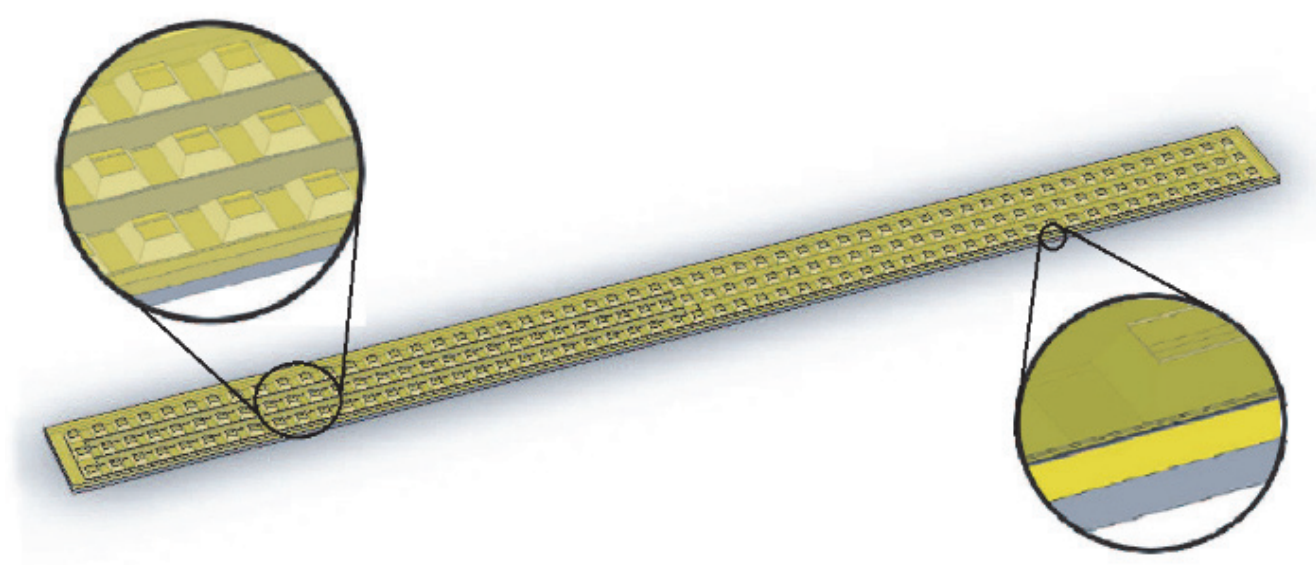

(a)

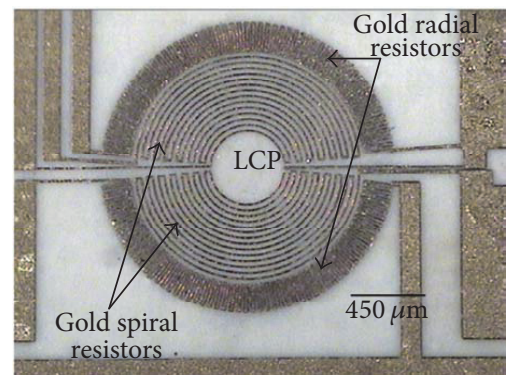

(b)

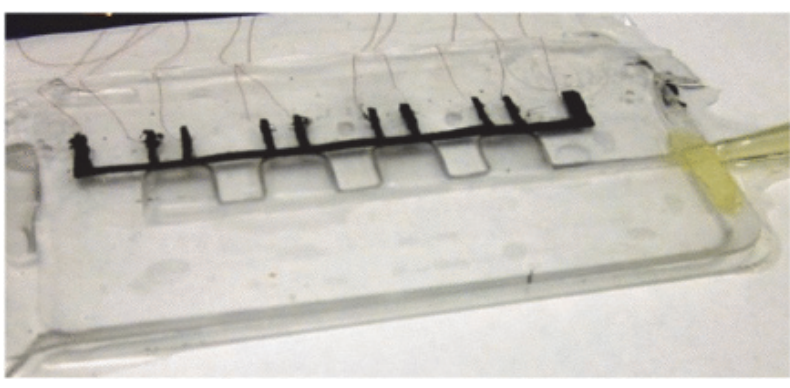

(c)

FIGURE 4: Summary of piezoresistive ALL pressure sensors. (a) High-density array of pressure sensors introduced by Fernandez et al. [13]. (b) Flexible arrays of polymer MEMS pressure-gradient sensors developed by Kottapalli et al. [14]. (c) Flexible ALL pressure sensor array presented by Yaul et al. [15].

(Figure 4(a)). A strain gauge mounted on a flexible substrate functioned as the sensing element, which was a $20 \mu \mathrm{m}$ thick silicon layer placed at the edges of a square silicon cavity that had a width of $2000 \mathrm{um}$. AUV s could detect and locate nearby obstacles and optimize navigation by measuring the pressure fluctuations acting on a vehicle surface in unsteady flow with a high-density pressure sensor array.

To yield an enhanced performance in underwater surveillance, Kottapalli et al. [14] developed flexible arrays of polymer MEMS pressure-gradient sensors, which could be conveniently installed on the curved surfaces of AUV bodies. The sensor consisted of a liquid crystal polymer (LCP) membrane deposited with gold piezoresistors (Figure 4(b)). The LCP membrane material offered high robustness and sensitivity compared with silicon-based hair vertical structures or thin metal cantilever beams. A Cr $(20 \mathrm{~nm}) / \mathrm{Au}$ $(700 \mathrm{~nm})$ thick gold layer was sputtered to integrate the devices on a flexible substrate. The metal media on $100 \mu \mathrm{m}$ thick LCP were patterned to form electrical connection for flexible backing.

For underwater sensing, sensors are required that exhibit a high pressure sensitivity in a certain range of water fluctuation. Yaul et al. [15, 34] presented a flexible ALL pressure sensor array (Figure 4(c)). Each sensor consisted of a PDMS diaphragm and a resistive strain gauge containing a composite of conductive carbon black-PDMS. Four sensors with an interval of $15 \mathrm{~mm}$ were arranged in a linear array, and the dynamic response model was established.

Table 1 provides a summary of the piezoresistive ALL sensors. Sensors with a vertical hair are mainly sensitive to flow velocity, and flat ALL sensors are mainly sensitive to pressure [13-15].

3.2. Piezoelectric ALL Sensors. Piezoelectricity is the electric charge that accumulates in certain solid materials in response to applied mechanical stress. Power supply is needed for piezoresistive or metal strain gauge to tie the sensor in a Wheatstone bridge. The flow stimulus acting on the sensing element would generate a voltage output. But sensors on the basis of the piezoelectric effect do not require a power supply. Table 2 provides a summary of piezoelectric ALL sensors.

For bioinspired underwater sensing, Asadnia et al. [16] fabricated and characterized a piezoelectric sensor array made of $\mathrm{Pb}(\mathrm{Zr0}$.52Ti0.48) O3 thin film (Figure 5(a)). A floating-bottom electrode was designed to detect water motion with low frequency. The sensors were arranged in a $2 \times 5$ array with gold interconnections on a flexible LCP substrate. They were self-powered and did not require an external power supply, which was advantageous to reduce the bulky load of power supplies on underwater vehicles.

In 2015, the same group [17] fabricated flexible and surface-mounted arrays of MEMS ALL sensors with 
TABLE 1: Summary of piezoresistive ALL sensors.

\begin{tabular}{|c|c|c|c|}
\hline Author & Size & Sensitivity & Other parameters \\
\hline Fan et al. [8] & $\begin{array}{c}\text { In-plane cantilever: } 1100 \mu \mathrm{m}(l), \\
180 \mu \mathrm{m}(w), 17 \mu \mathrm{m}(t) \\
\text { Vertical cilium: } 820 \mu \mathrm{m}(h), 100 \mu \mathrm{m} \\
(w), 10 \mu \mathrm{m}(t)\end{array}$ & $100 \mathrm{~mm} \mathrm{~s}^{-1}$ & Hair method: PDMA \\
\hline $\begin{array}{l}\text { Yang et al. } \\
{[10,32,33]}\end{array}$ & $\begin{array}{c}\text { Beam cilium: } 2 \mu \mathrm{m}(t), 40 \mu \mathrm{m}(w) \\
100 \mu \mathrm{m}(l) \\
\text { Cilium: } 80 \mu \mathrm{m}(d), 500 \mu \mathrm{m}(h)\end{array}$ & $0.1 \mathrm{~mm} \mathrm{~s}^{-1}$ & $\begin{array}{l}\text { Hair method: PDMA } \\
\text { Deflection: } 55^{\circ}\end{array}$ \\
\hline McConney et al. [11] & $\begin{array}{c}\text { Microfabricated hair: } 550 \mu \mathrm{m}(l) \\
100 \mu \mathrm{m}(d) \\
\text { Total height: } 825 \mu \mathrm{m}\end{array}$ & $75 \mu \mathrm{m} \mathrm{s}^{-1}$ & $\begin{array}{l}\text { Hair method: } \\
\text { photopolymerization }\end{array}$ \\
\hline Qualtieri et al. [12] & $\begin{array}{c}\text { Cantilever: } 100 \mu \mathrm{m}(w), 200 \mu \mathrm{m} \text { to } \\
600 \mu \mathrm{m}(l) \\
\text { Piezoresistor: } 50 \mu \mathrm{m}\end{array}$ & Not available & $\begin{array}{l}\text { Hair method: } \\
\text { micromachining }\end{array}$ \\
\hline Yaul et al. $[15,34]$ & $10 \mathrm{~mm}(d), 1 \mathrm{~mm}(t)$ & Not available & $\begin{array}{c}\text { Pressure resolution: } \\
1.5 \mathrm{~Pa} \\
\end{array}$ \\
\hline Fernandez et al. [13] & $\begin{array}{c}\text { Diaphragm size: } \sim 1 \mathrm{~mm} \\
\text { Spacing: } \sim 1 \mathrm{~mm} \\
\text { Thickness: } \sim 1 \mathrm{~mm} \\
\end{array}$ & $1 \mu \mathrm{V} / \mathrm{Pa}(10 \mathrm{~V})$ & Pressure resolution: $1 \mathrm{~Pa}$ \\
\hline Kottapalli et al. [14] & $330 \mu \mathrm{m}(t), 2 \mathrm{~mm}(d)$ & $\begin{array}{c}14.3 \mu \mathrm{V} / \mathrm{Pa} \text { (pressure) } \\
90.5 \mathrm{mV} / \mathrm{ms}^{-1} \\
\text { (velocity) }\end{array}$ & $\begin{array}{l}\text { Flow resolution: } \\
\qquad 25 \mathrm{~mm} \mathrm{~s}^{-1}\end{array}$ \\
\hline
\end{tabular}

$l$ : length; $w$ : width; $t$ : thickness; $d$ : diameter; $h$ : height.

TABLE 2: Summary of piezoelectric ALL sensors.

\begin{tabular}{lccc}
\hline Author & Size & Material & Parameters \\
\hline Asadnia et al. [16] & $2 \times 2 \mathrm{~mm}$ & $\mathrm{~Pb}($ Zr0.52Ti0.48) O3 & $\begin{array}{c}\text { Resolution: } 3 \mathrm{~mm} \mathrm{~s}^{-1} \\
\text { Sensitivity: }\end{array}$ \\
& $1.4 \times 1.4 \mathrm{~mm}$ & Si60 hair $22 \mathrm{mV} /\left(\mathrm{mm} \mathrm{s}^{-1}\right)$ \\
Abdulsadda and Tan [35] & $8 \mathrm{~mm} \times 2 \mathrm{~mm} \times 200 \mu \mathrm{m}$ & IPMC & Threshold detection limit: $8.2 \mu \mathrm{m} \mathrm{s}^{-1}$ \\
\hline
\end{tabular}

versions of SNs and CNs based on the piezoelectric sensing principle (Figure 5(b)). The sensors were optimized with a stereolithographically fabricated polymer hair cell mounted on the microdiaphragm with a floating-bottom electrode. Canal-inspired systems contained a canal made of polymer; there were pores on surfaces of the canal that guided outside flows to the ALL sensors located inside the canal. CNs could reduce the interference generated by low-frequency steady flows and substantially filtered out oscillatory flows below $10 \mathrm{~Hz}$.

Abdulsadda et al. [18, 35, 50-52] exploited the inherent sensing capability of ionic polymer-metal composites (IPMCs) to develop ALL sensors (Figure 5(c)). The composites consisted of three layers and an ion-exchange polymer membrane placed between the metal electrodes. Inside the polymer, anions covalently fixed to polymer chains were balanced by positively charged mobile cations. Polarization induced by deformation of the lamella produced a detectable electric output that could be proportional to the bending amplitude of the vertical hair.
3.3. Capacitive ALL Sensors. Capacitive detection principles have advantages of high sensitivity and low power consumption, which are a new method to imitate sensing hairs found on the cerci of crickets. Capacitive sensor is a transforming system that can convert hydrodynamic excitation into a capacitance change [3].

Inspired by the acoustic flow-sensitive hairs found on the cerci of crickets, Krijnen et al. [19, 23, 53] utilized sacrificial polysilicon technique and SU8 polymer processing technique to fabricate silicon nitride-suspended membranes and tiny hairs with diameters of $50 \mathrm{um}$ to $1 \mathrm{~mm}$ (Figure 6(a)). There were thin chromium electrodes on the top of the membranes to form variable capacitances, and a capacitive readout was provided by the substrate. The filiform hairs had a high sensitivity close to thermal threshold and were perceptive to low-frequency sound. Dagamseh et al. [12, 54] assessed the ability of this sensor array to detect distributions of flow velocity generated by a vibrating sphere and investigated the influence of various source parameters on object distance measurement. 


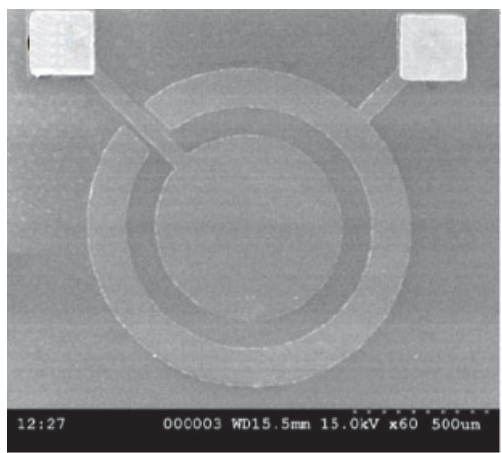

(a)

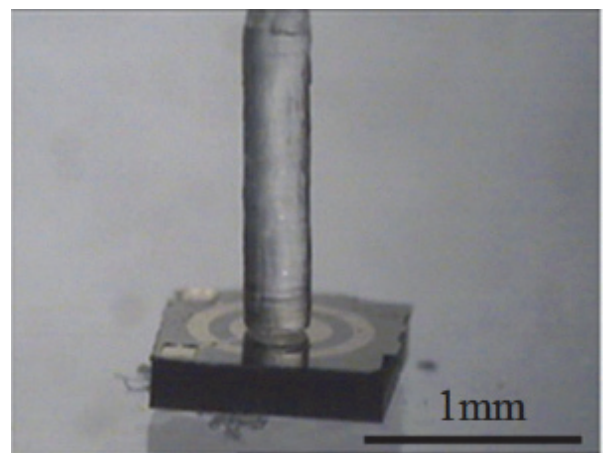

(b)

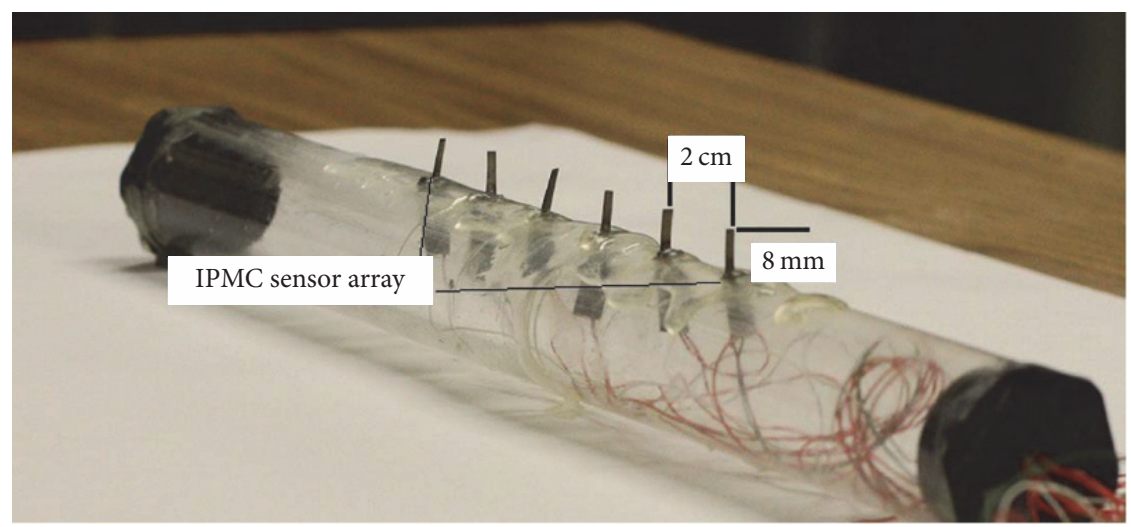

(c)

Figure 5: Piezoelectric ALL sensors. (a) Thin-film piezoelectric pressure sensor arrays fabricated by Asadnia et al. [16]. (b) Flexible and surface-mountable arrays of MEMS ALL sensors developed in 2015 [17]. (c) IPMCs ALL sensors developed by Abdulsadda and Tan [18].

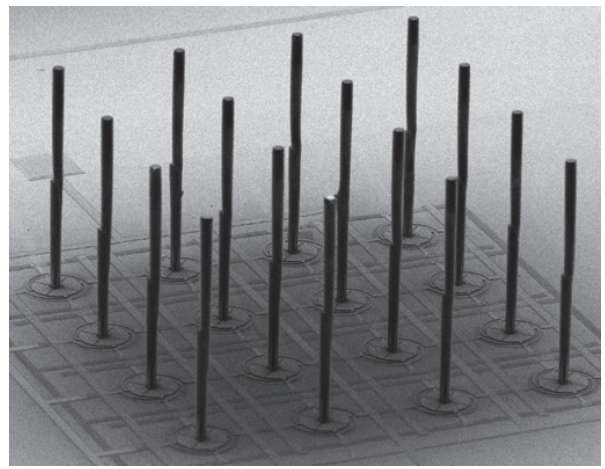

(a)

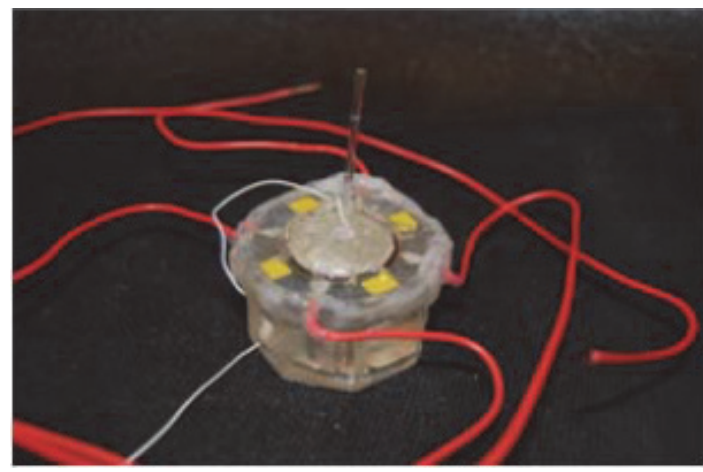

(b)

Figure 6: Capacitive ALL sensors. (a) Capacitive ALL hair sensors fabricated by Krijnen et al. [19]. (b) Capacitance-based whisker-like artificial sensor designed by Stocking et al. [20].

Inspired by seal vibrissae, Stocking et al. [20] designed a capacitive whisker-like sensor for measuring flow velocity and orientation (Figure 6(b)). The sensors were parallel plate capacitors covered by PDMS membranes separated into four distinct quadrants and a rigid artificial whisker mounted on the top of the cone-in-cone base. Numerical modeling predicted the capacitive output signals within a range of $1 \mathrm{pF}$ when flow velocity ranged from 0 to $1.0 \mathrm{~m} / \mathrm{s}$.
3.4. Optical ALL Sensors. Optical sensing principles are explored to fabricate ALL sensors. Klein and Bleckmann [21] developed ALL canals equipped with optical flow sensors. They could detect the water movements generated by a static vibrating sphere, the periodic turbulence caused by an upstream cylinder, or the water fluctuations caused by a passing object (Figure 7(a)). Transparent silicone bars were fabricated and installed inside canal segments. An infrared 


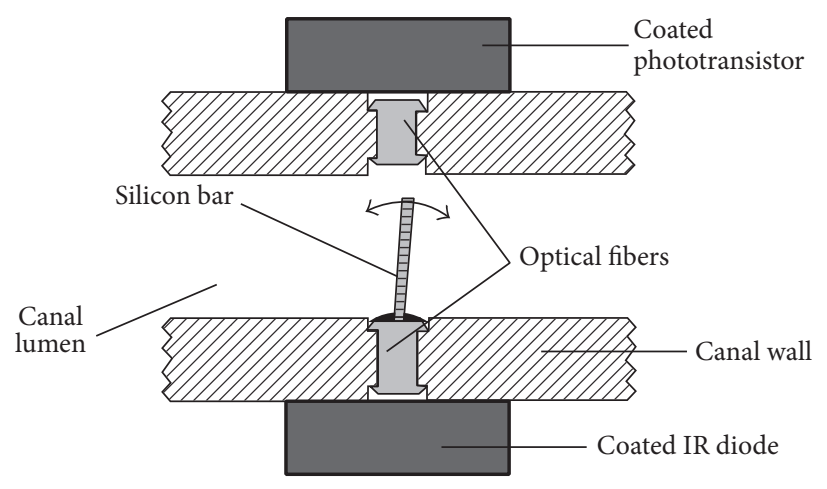

(a)

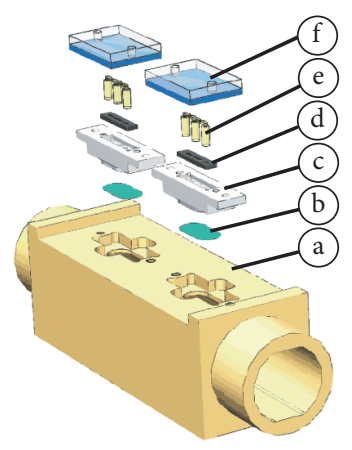

(b)

FIgURE 7: Optical ALL sensors. (a) ALL canals equipped with optical flow sensors developed by Klein and Bleckmann [21]. (b) ALL canals developed by Herzog et al. [22].

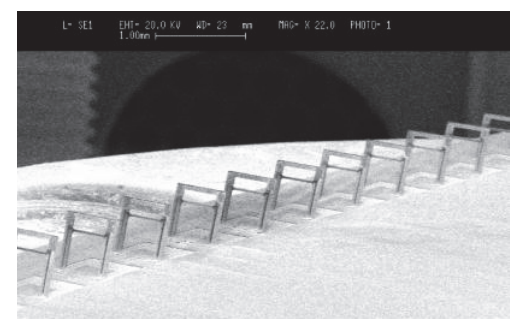

(a)

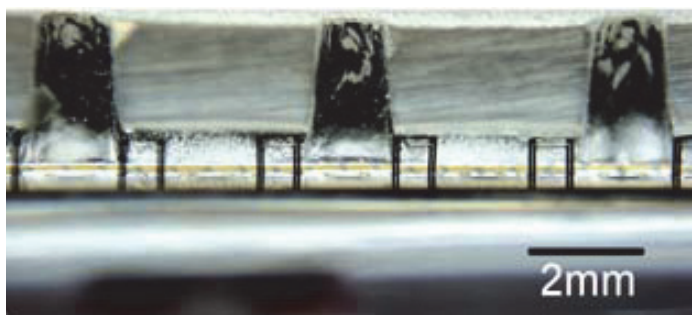

(b)

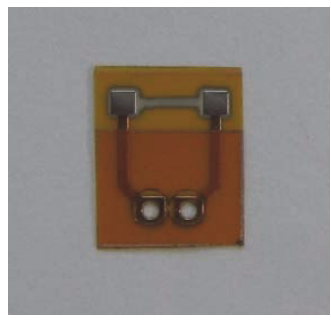

(c)

Figure 8: Hot-wire ALL sensors. (a) Hot-wire ALL sensors developed by Yang et al. [23]. (b) ALL sensor array in a canal [24]. (c) Novel micromachined hot-film ALL sensor presented by Liu et al. [25].

light-emitting diode was illuminated at the end of each silicone bar to detect the bending of the bar. An optical fiber with a SMD phototransistor was illuminated at the opposite end of the silicone bar, and the output was amplified. The output of transistor was fed to an analog-to-digital converter (ADC) and transferred to the computer to store. The pressure difference between the pores on the canal surface led to a fluid flow inside the ALL canals, which in turn made the silicone bars deflected.

In the same institution, Herzog et al. [22] employed the ALL canals to estimate bulk flow speed by the spatiotemporal propagation of flow undulations. They optimized the design in modular form (Figure 7(b)) for the purpose of industrial applications of flow measurement in tap water. Finite element method was utilized to analyze the flow characteristics inside the canal system and the relationship between the flow and canal dimensions. Through a flexible membrane, the sensors were separated from the main stream. Experiment showed that the ALL canals were able to sense flow fluctuations.

3.5. Hot-Wire ALL Sensors. Hot-wire anemometers take advantage of a thin wire electrically heated above ambient temperature. Since the resistance of most metals is proportional to temperature, while water flowing past the wire causes a cooling effect on it, a relationship between the resistance of the metal and the flow velocity can be obtained. Such relationship is used to measure wind speed and can be manufactured at low cost and high efficiency; accordingly, researchers have explored it to develop ALL sensors.

In 2006, Yang et al. [55] developed a surfacemicromachined, out-of-plane ALL sensor array using the principle of thermal HWA (Figure 8(a)), which exhibited high sensitivity, small dimensions, and low interference of flow field. The hot wire was elevated above the substrate by two prongs, similar to SNs in fish. First, photolithography technique was utilized to make the sensor in plane and then a 3D magnetic assembly method was employed to assemble it out of plane. Photolithography defined the resultant elevation that corresponded to the design length of the prongs. Owing to the photolithography technique, the micromachined HWA sensors could have an actual prong length ranging from $50 \mu \mathrm{m}$ to $2 \mathrm{~mm}$. Inspired by the CNs of fish, Chen et al. [24] put the ALL sensor array into a canal to track the distance and position of a vibrating dipole source (Figure 8(b)). Pandya et al. [56] implemented an algorithm that assisted in underwater positioning and tracking for vibration dipole source.

Liu et al. [25] presented a new micromechanical hot-film ALL sensor which was implemented by film-deposition process combined with a standard printed circuit (Figure 8(c)). The sensing element consisted of $\mathrm{Cr} / \mathrm{Ni} / \mathrm{Pt}$ with a temperature coefficient of resistance of $2000 \mathrm{ppm} / \mathrm{K}$. It was preprinted on a flexible printed circuit board by magnetron sputtering or pulsed laser deposition. The sensors were easy to attach on 


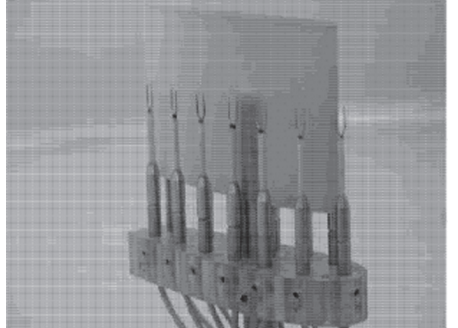

(a)

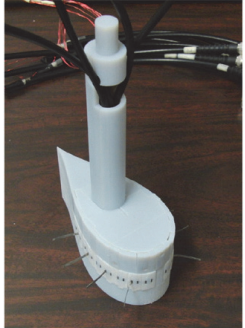

(b)

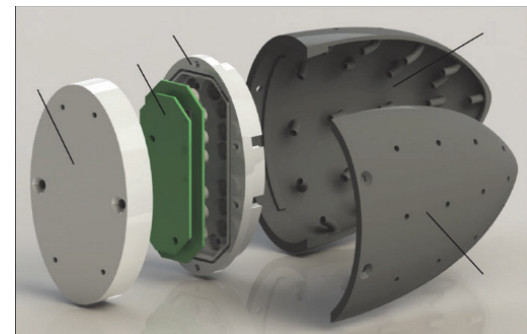

(c)

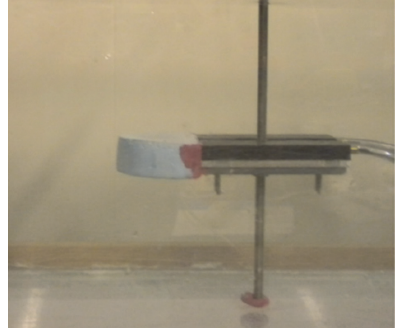

(d)

FIgure 9: Passive ALL systems. (a) ALL systems invented by Pandya et al. [26] with both micromachined and commercial HWA sensors. (b) Robot designed by DeVries et al. [27]. The robot prototype was a 3D-printed 2D-airfoil shape. (c) 3D fish-shaped head developed by Chambers et al. [28]. (d) ALL system with a rigid body developed by Venturelli et al. [4].

the surface of objects, because they had good mechanical properties and sensing capabilities, simple structure, and flexibility.

\section{Artificial Lateral Line System}

In the previous section, the sensing principles, design methods, fabrication technologies, and response characteristics of ALL sensors were introduced. This section focuses on the application of ALL to robot fishes and AUVs. According to their structure, ALL systems can be divided into rigid carrier and robotic fish carrier. The robotic fish carrier is a bionic robot fish that can swim with the tail fin and is instrumented with pressure sensors to detect hydrodynamic stimuli. The rigid carrier is a sensor platform with a rigid body that cannot move by itself. With different outline shapes, structures, and sensor distributions, ALL systems can measure different hydrodynamic parameters and achieve biomimetic underwater vehicle auxiliary navigation control.

\subsection{Structure of ALL Systems}

4.1.1. Rigid ALL Carrier. The following ALL system is a sensor platform with a rigid body and a forced-to-fixed orientation. This ALL system is mainly applied to underwater flow sensing for AUV control.

Inspired by the fish lateral line, Pandya et al. [26] invented ALL systems equipped with both micromachined and commercial HWA sensors. There were two experimental geometries to form different sensor arrangements and hydrodynamic acquisitions. The first was a linear arrangement consisting of 16 HWAs. The second was a solid airfoil mimicking the appearance of a fish, which consisted of 13 HWAs (Figure 9(a)). They successfully caught the dipole source when the source diagonally passed through the linear geometries.

Bionic sensing method improved the ability of AUVs to navigate and respond to their environment. DeVries et al. [27] designed and constructed a robot to experimentally demonstrate the distributed flow sensing and closed-loop control strategies. 3D-print technology was utilized to fabricate the 2D-airfoil robot prototype which was characterized by Joukowski mapping parameters and extruded in the vertical direction (Figure 9(b)). To minimize fluid effects, pressure sensors were mounted above the IPMC sensor area.

For sensing the fluid interactions that occur, Chambers et al. [28] developed an ALL system with a 3D fish-shaped head to investigate the pressure response to hydrodynamic excitation (Figure $9(\mathrm{c})$ ). There were 33 pressure taps arranged on the head surface, which mimicked a natural system, and another 33 sensors were attached to the corresponding taps by rubber tubing.

To better understand the sensing environment, Venturelli et al. [4] developed an ALL system with a rigid body and curved head (Figure 9(d)). The pressure sensors were distributed parallel on both sides. The visualization of the flow regime was first achieved by digital particle image velocimetry and then the local measurements were obtained by the ALL system.

4.1.2. Robotic Fish ALL Carrier. A robotic fish carrier is a robotic fish with a tail fin. It is equipped with pressure sensors to detect hydrodynamic stimuli. This ALL carrier is mainly applied to research the control strategy and moving characteristic of a robot fish.

To solve the problem of sensing surrounding hydrodynamic environment in underwater robots, Li et al. [29] designed an ALL system for a robotic fish with nine pressure sensors (CPS131) (Figure 10(a)). Three of the sensors were located in front of the fish head, while the other six were symmetrically distributed in the left and right sides. The pressure sensors were welded on the printed circuit board and embedded inside the robotic fish, leaving the sensing chip in contact with the underwater world.

For the navigation with hydrodynamic assistance of bionic propulsion, Salumäe and Kruusmaa [30,36] utilized the FILOSE robot fish equipped with five piezoresistive pressure sensors (MS5407-AM) to detect hydrodynamic stimulus and control the robot motion with respect to the fluid environment (Figure 10(b)). The sensors were installed inside the rigid plastic body and connected to the outside through pressure taps. A total of five pressure taps on the head surface were separately distributed at the nose tip, on both sides $50 \mathrm{~mm}$ and $100 \mathrm{~mm}$ from the nose. 


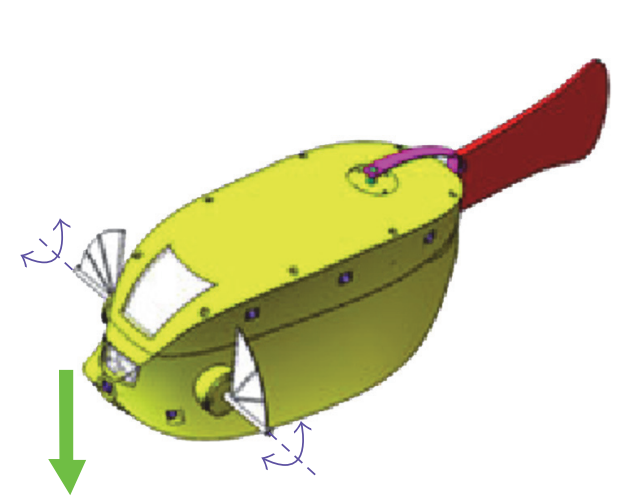

(a)

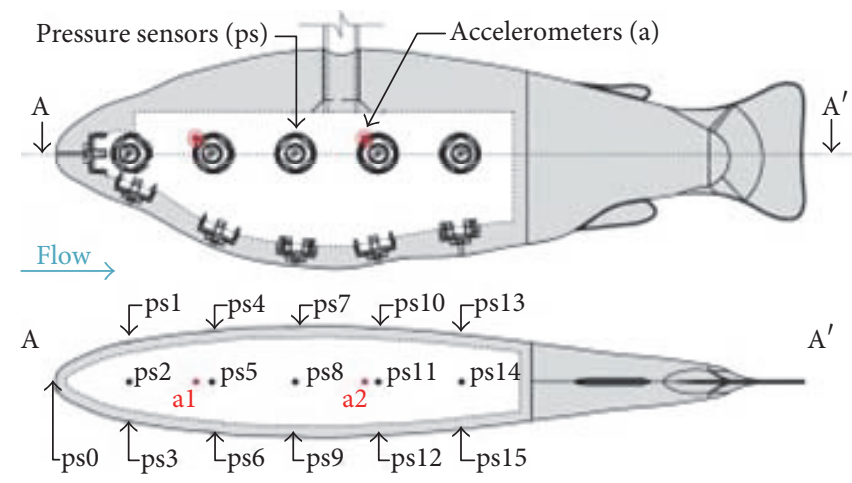

(c)

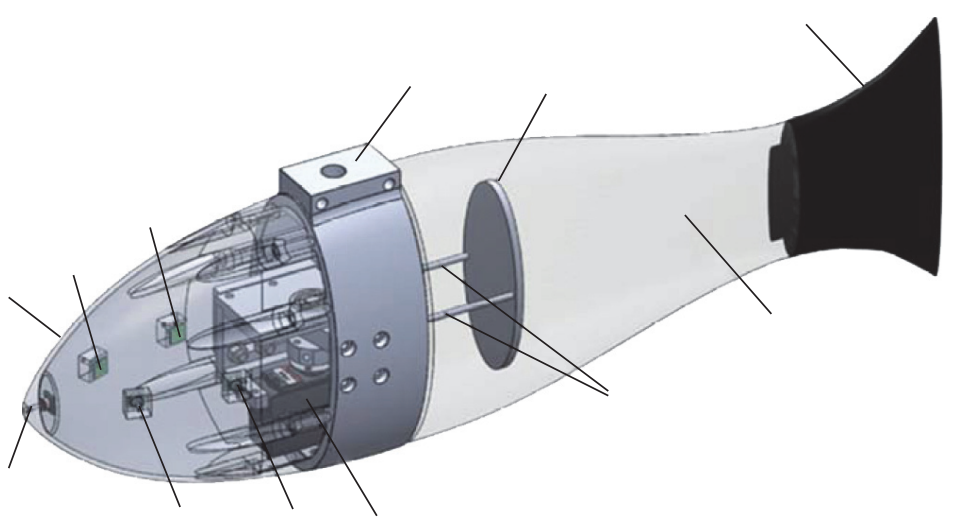

(b)

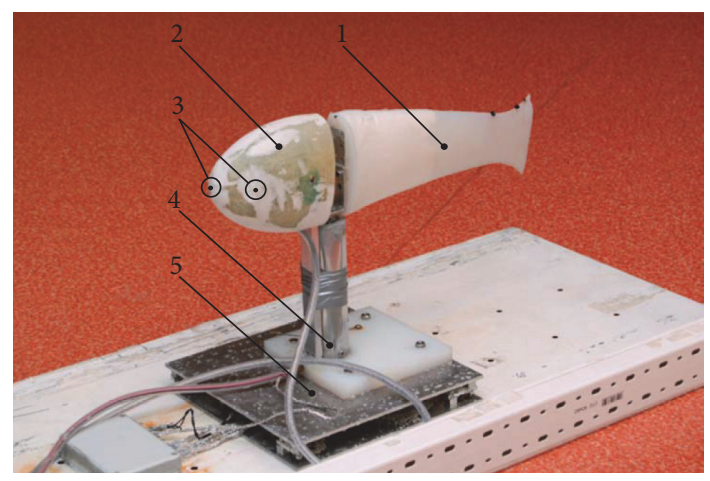

(d)

FIgURE 10: Active ALL system. (a) ALL system for a robotic fish designed by Li et al. [29]. (b) FILOSE robot fish equipped with five pressure sensors [30]. (c) ALL system developed by Fuentes-Pérez et al. [5]. (d) Sensing-actuation coupling of a robotic trout described by Kruusmaa et al. [31].

Fuentes-Pérez et al. [5] developed an ALL system with a shape of a plastic-shell rainbow trout (Figure 10(c)). The ALL platform was equipped with 16 pressure sensors (SM5420C030-A-P-S) with sensitivity over 0 to $207 \mathrm{kPa}$ and two 3 -axis accelerometers (ADXL325BCPZ). The ALL system aimed to create an algorithm for a convenient underwater flow velocity estimation.

For the speed control strategy, Kruusmaa et al. [31] developed a sensing-actuation robotic trout that detected the fluctuations of flow velocity through pressure sensor and adjusted its tail-beat frequency to remain stationary in the uniform flow (Figure 10(d)). They used the same pressure sensors as Salumäe. The pressure sensor signal was transmitted to 18-bit ADC with 8-fold gain. The sensor sensitivity was $3.57 \mathrm{~Pa}$ with the ADC reference voltage of $2048 \mathrm{~V}$. All the electronic components were installed into a waterproof bag and placed in the head of the robotic fish. Table 3 summarizes the achievement of the ALL system.

4.2. Hydrodynamic Detection. Researchers have developed ALL systems for underwater hydrodynamic field measurement to achieve similar performance between artificial equipment and real fish. Yang et al. [10] developed a beamforming algorithm that allowed the ALL sensor array to describe realistic hydrodynamic events in a 3D domain, which was also successfully applied for ALL system in [25]. Different from the linear ALL sensor array, the sensors in ALL system are distributed along the streamline body surface with symmetrical arrangement, which is in favor of detecting the hydrodynamic environment. The detection of underwater flow fields includes flow regime discrimination, velocity estimation, orientation detection, and object identification. With the sensing signal and mathematical analysis adopted by ALL system, the vehicle control strategy is summarized to improve the maneuvering efficiency.

4.2.1. Flow Regime Discrimination. The two basic fluid states, namely, uniform flow and periodic turbulence, are considered. The periodic turbulence means the regular periodic vortices shedding from an object in uniform flow, called a Karman vortex street (KVS). This fluid phenomenon can be reproduced in the laboratory setting. The discrimination of KVSs from uniform flows can be achieved by analyzing the magnitude of fluctuations and dominant frequency [36].

Kottapalli et al. [14] studied the flow regime discrimination based on the turbulence intensity and sensor consensus dominant frequency. The average turbulence density in the KVS was estimated to be $30 \%$ higher than in the uniform flow. More than half of the sensors simultaneously detected the vortex shedding frequency (VSF) as their primary frequency. 
TABLE 3: Summary of ALL systems.

\begin{tabular}{|c|c|c|}
\hline Author & Sensors & Achievements \\
\hline Pandya et al. [26] & 16 HWAs & $\begin{array}{l}\text { To visualize an underwater flow fluctuation } \\
\text { using adaptive beamforming together with } \\
\text { ALL systems. }\end{array}$ \\
\hline DeVries et al. [27] & 8 IPMC sensors and 4 pressure sensors & $\begin{array}{c}\text { To present estimation and control strategies } \\
\text { enabling an underwater vehicle to assimilate } \\
\text { measurements from an ALL system and } \\
\text { estimate flow parameters for feedback } \\
\text { control. }\end{array}$ \\
\hline Chambers et al. [28] & 33 pressure sensors & $\begin{array}{l}\text { To investigate fluid interactions with } \\
\text { underwater vehicles. The periodic vortexes } \\
\text { are formed behind cylinders with different } \\
\text { diameters }(2.5,4.5 \text {, and } 10 \mathrm{~cm}) \text { and flow } \\
\text { velocities }\left(9.9,19.6 \text {, and } 26.1 \mathrm{~cm} \mathrm{~s}^{-1}\right) \text {. }\end{array}$ \\
\hline Venturelli et al. [4] & 20 pressure sensors & $\begin{array}{l}\text { To present a depth understanding of } \\
\text { hydrodynamic characteristics in uniform } \\
\text { flow and Karman vortex streets. }\end{array}$ \\
\hline Li et al. [29] & 9 pressure sensors (CPS131) & $\begin{array}{l}\text { To measure the swimming speed of a } \\
\text { robotic fish and make a fast control for it. }\end{array}$ \\
\hline Salumae et al. [36] & 5 pressure sensors (MS5407-AM) & $\begin{array}{l}\text { To identify and discriminate flow regimens, } \\
\text { detect the orientation with respect to the } \\
\text { flow direction, measure the flow speed, and } \\
\text { estimate the robot position in the wake of an } \\
\text { object. }\end{array}$ \\
\hline Fuentes-Pérez et al. [5] & 16 pressure sensors (SM5420C-030-A-P-S) & $\begin{array}{l}\text { To propose an algorithm that allows the ALL } \\
\text { systems to estimate current flow velocity } \\
\text { without sensor calibration. }\end{array}$ \\
\hline Kruusmaa et al. [31] & 2 pressure sensors & $\begin{array}{l}\text { To detect changes in the laminar flow speed } \\
\text { and adjust its tail-beat frequency for steady } \\
\text { swimming. }\end{array}$ \\
\hline
\end{tabular}

Chambers et al. [28] proposed that the instantaneous pressure difference between the nose and surrounding sensors can be an indicator in a turbulent flow at low cruising velocity. A turbulence intensity measurement with a short time window $(0.2 \mathrm{~s})$ was verified in this proposal. The turbulence intensity peak is clearly visible in the center of the region from the pressure data.

4.2.2. Velocity Estimation. The main velocity estimation method is the Pitot equation, which is derived from the Bernoulli equation. This method requires the pressure drop between the stagnation point $\left(P_{1}\right.$, static pressure) and the second point pressure $\left(P_{2}\right.$, dynamic pressure) on the body surface in the free-stream flow. When the system is aligned to the oncoming flow, the pressure sensor on the tip of the system nose acquires the stagnation pressure, while the lateral sensors measure the dynamic pressure. A semiempirical correction factor $\left(\beta_{U}\right)$ must be applied to the equation because the side sensors are under both static and dynamic pressure. Another method uses only the average pressure on the robot sides. The relationship between the average pressure difference and the flow velocity is well fitted with a polynomial equation [36]:

$$
\frac{\rho U^{2}}{2}=\left(P_{1}-P_{2}\right)=\Delta P_{1,2} \longrightarrow
$$

$$
U=\sqrt{\frac{2 \cdot \beta_{U} \cdot \Delta P_{1,2}}{\rho}},
$$

where $\rho$ is the water density and $U$ is the flow velocity.

Although the above method could directly estimate the flow velocity by calculating pressure difference, the sensors need to be typically calibrated before each measurement. Fuentes-Pérez et al. [5] proposed a two-stage signal processing method that did not require sensor calibration. First, a frequency domain analysis of pressure oscillation was conducted over a sampling interval of a few seconds. Second, a resample estimate with the Bernoulli relation was conducted at the original acquisition rate.

For an ALL with a rigid body, under the uniform flow of a certain velocity, the magnitude of the VSF in the KVS can be an indicator to estimate the flow velocity [28]. However, the flow velocity can only be retrieved from the VSF if the cylinder size is known, which is not the case in an autonomous robot moving in a current. Chagnaud et al. [57] suggested the cross-correlation principle. Two flexible micropillars separated in the downstream direction were utilized to measure the bending motion induced by flow. A cross-correlation of their bending motions provided an 


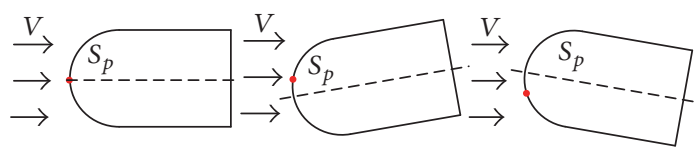

FIGURE 11: Flow direction detection. $S_{p}$ is the stagnation point, and $V$ is flow velocity.

accurate estimation of the flow velocity vector in the direction of the micropillars.

4.2.3. Flow Direction Detection. ALL system can determine the orientation of the system with respect to the oncoming flow. When the platform is parallel to the incoming flow, the stagnation point is located in the symmetry points of the head; therefore, the stagnation point can be adopted for direction judgments (Figure 11).

Venturelli et al. [4] analyzed the pressure difference on both sides of a submarine to calculate the deflection angle of the submarine. The mean value of the spatiotemporal pressure measured by each array could be an indicator. When the platform was aligned to the flow, it was equal, while when the platform was angled with respect to the flow, it was different. The pressure difference was proportional to the angle.

Salumae et al. [36] conducted a comparative analysis to identify the direction and position of the platform, which was the same principle as that of Venturelli et al. [4]. A linear relationship between pressure difference and robot orientation was obtained by a separate analysis of the voltage output of the anterior and posterior sensor pair. The slope of the pressure difference was proportional to flow velocity.

4.2.4. Object Identification. ALLs can localize vibrating biotic and abiotic sources. By applying adaptive beamforming (Capon's method), Liu et al. [25] visualized underwater flow disturbances, including acoustic dipoles source. The error of imaging dipole sources was less than $10 \%$. A fluid vibration caused by a human finger also can be detected by ALL sensors.

Dagamseh et al. [23] took the VSF magnitude as a predictor to identify the source feature. In the area directly behind the cylinder, the sensor platform position in the suction zone and vortex formation zone could be estimated by the magnitude reduction of VSF. It was an indicator to track the platform in the downstream of the cylinder and a unique predictor for KVS identification.

4.2.5. Robot Control Strategy. Scholars have developed different control methods for AUV and robot fish to improve the maneuvering efficiency.

DeVries et al. [27] presented an estimation of flow properties and feedback control strategies for AUV. They used the potential flow theory to model the flow characteristics behind the foil in the uniform flow and past an obstacle. The estimation of free flow velocity, angle of attack, and relative position of an upstream obstacle was obtained by theoretically justified nonlinear estimation. The estimated flow properties were utilized to execute bionic behavior, including rheotaxis and station holding. Kruusmaa et al. [31] derived a linear control law for an artificial fish using the linear relationship between the tail-beat frequency and swimming speed. They achieved an experiment on controlling the swimming speed by an on-board pressure sensing system. Salumae et al. [36] demonstrated a navigation way of reducing the energy consumption of an underwater vehicle. In the periodic turbulence flow, staying at the station behind an object can reduce the energy consumption, because it was the reduced flow zone in the wake of an object.

\section{Research Trends and Existing Problems}

In the previous sections, we introduced ALL sensors and their applications to robot fishes, which provided the basic objective to understand the ALL research status. At present, ALL sensors cannot match the capability of natural lateral line organs. For further research, many problems remain unsolved and unexplored.

For the theoretical model, there are still some problems, such as the synchronization of acquisition system, conduction efficiency between fluid and sensors, and optimum frequency response. For ALL sensors, the piezoresistive effect is the most widely used, which could achieve the highest sensitivity of $75 \mu \mathrm{m} \mathrm{s}^{-1}$ [11], but the piezoresistive materials are sensitive to temperature change, thus requiring strict calibration before being employed. The readout mechanism of capacitive ALL sensor can provide high resolution with low power consumption, but water is not the appropriate conductive medium for it because of the electrical insulation of the electrodes. To manufacture ALL sensors with sensitivities equivalent to the natural system, microfabrication techniques need to be improved. With the stringent environmental requirements, the ALL sensor application is far from batch production and stuck in the laboratory.

From the aspect of energy harvesting and self-power, piezoelectric sensors are the most advantageous. Asadnia et al. [16] developed a piezoelectric sensor array that was selfpowered and did not require an external power supply. By utilizing specific flows rather than contending with them, AUV can reduce energy consumption and improve the maneuvering capabilities. For underwater sensing, sensors are required to bond with the soft surface similar to fish. Thus, many groups have developed flexible ALL sensor arrays to realize the goal. For instance, Yaul [34] presented a flexible pressure sensor array based on the piezoresistive effect, Asadnia et al. [16] fabricated flexible piezoelectric sensor arrays that were surface-mountable, and Liu et al. [25] presented a novel micromachined hot-film flow sensor system. In this respect, the ALL systems that navigate autonomously most likely feature a soft body similar to fish.

For ALL array, the distribution method continues to require a thorough investigation because many sensors present a regular linear distribution currently. Improving the gathering of meaningful hydrodynamic information is important. From a bionics perspective, the inner connection of sensor space, sensitivity and system topology distribution, 
and detection efficiency should be determined to clarify whether different kinds of sensors need distinct intervals.

For the algorithms based on ALL, effort should be made to compensate for the error and excursion caused by the electronic and mechanical structure through the subsequent signal processing and calculation model. A real-time neural network algorithm or fast Fourier transform may help. For the long integration time in laboratory measurements, considering that a fish robot in many cases has to react rapidly to a hydrodynamic stimulus, problems of error accumulation present a crucial issue, which has not been addressed thus far. New algorithms must be proposed to ensure that ALL could be accurate in different flow environments for a certain time. Data processing should be transferred from the offline computer to a real-time chip, and how to generate meaningful responses rapidly in an unsteady hydrodynamic environment should be considered.

In the future, ALL will have broad development prospects and promotion space, the application of ALL will enlarge, and it will make the underwater vehicles more autonomic and intelligent. With a similar perception to real fish, ALL can be used in military, underwater search and rescue, navigation, and environment monitoring. For AUV control, ALL can help increase the maneuvering efficiency and enable AUVs to perform various tasks, including localizing and avoiding obstacles as well as navigating in narrow spaces.

\section{Conclusion}

In this paper, we reviewed ALL, including the biophysics research, sensor fabrication, and application to robot fishes. Various ALL sensors based on different sensing modalities were introduced. Most of the sensors are in micron level with the development of the MEMS technology and PDMA method. The piezoresistive sensors achieve the highest sensitivity of $75 \mu \mathrm{m} \mathrm{s}^{-1}$. The piezoelectric sensors are self-powered and have high signal-to-noise ratio and wide frequency band. The readout mechanism of capacitive ALL sensors can offer high resolution and low power consumption. They show an excellent sensing performance in different aspects. As for the application in hydrodynamic detection, the turbulence intensity can be the analyzing basis to discriminate flow regime. Bernoulli's principle, VSF, and cross-correlation principle are effective methods to estimate flow velocity. The adaptive beamforming algorithm could image a dipole source. For a robot fish, tail-beat frequency and speed are linearized, which gives a method for autonomous navigation. ALL systems provide a new method to detect underwater fluid field, which are costless and flexible. With the advent of new materials and the development of microfabrication technology, the ALL will possess enhanced performance similar to real fishes.

\section{Competing Interests}

The authors declare that no competing interests exist regarding the publication of this paper.

\section{Acknowledgments}

This research was supported by the National Natural Science Foundation of China (Grant no. 61540010), Key Science and Technology Program of Shandong Province, China (Grant no. 2014GH Y115032), and People's Livelihood Science and Technology Plan of Qingdao (Grant no. 14-2-3-63-nsh).

\section{References}

[1] J. Mogdans and H. Bleckmann, "Coping with flow: behavior, neurophysiology and modeling of the fish lateral line system," Biological Cybernetics, vol. 106, no. 11-12, pp. 627-642, 2012.

[2] K. P. Maruska, "Morphology of the mechanosensory lateral line system in elasmobranch fishes: ecological and behavioral considerations," Environmental Biology of Fishes, vol. 60, no. 13, pp. 47-75, 2001.

[3] T. Shizhe, "Underwater artificial lateral line flow sensors," Microsystem Technologies, vol. 20, no. 12, pp. 2123-2136, 2014.

[4] R. Venturelli, O. Akanyeti, F. Visentin et al., "Hydrodynamic pressure sensing with an artificial lateral line in steady and unsteady flows," Bioinspiration \& Biomimetics, vol. 7, no. 3, pp. 36004-36015, 2012.

[5] J. F. Fuentes-Pérez, J. A. Tuhtan, R. Carbonell-Baeza et al., "Current velocity estimation using a lateral line probe," Ecological Engineering, vol. 85, pp. 296-300, 2015.

[6] S. Coombs and J. C. Montgomery, "The enigmatic lateral line system," in Comparative Hearing: Fish and Amphibians, pp. 319362, Springer, New York, NY, USA, 1999.

[7] A. Schmitz, H. Bleckmann, and J. Mogdans, "Organization of the superficial neuromast system in goldfish, Carassius auratus," Journal of Morphology, vol. 269, no. 6, pp. 751-761, 2008.

[8] Z. Fan, J. Chen, J. Zou, D. Bullen, C. Liu, and F. Delcomyn, "Design and fabrication of artificial lateral line flow sensors," Journal of Micromechanics and Microengineering, vol. 12, no. 5, pp. 655-661, 2002.

[9] J. Chen, Z. Fan, J. Zou, J. Engel, and C. Liu, "Two-dimensional micromachined flow sensor array for fluid mechanics studies," Journal of Aerospace Engineering, vol. 16, no. 2, pp. 85-97, 2003.

[10] Y. Yang, N. Nguyen, N. Chen et al., "Artificial lateral line with biomimetic neuromasts to emulate fish sensing," Bioinspiration \& Biomimetics, vol. 5, no. 1, Article ID 016001, 2010.

[11] M. E. McConney, N. Chen, D. Lu et al., "Biologically inspired design of hydrogel-capped hair sensors for enhanced underwater flow detection," Soft Matter, vol. 5, no. 2, pp. 292-295, 2009.

[12] A. Qualtieri, F. Rizzi, M. T. Todaro, A. Passaseo, R. Cingolani, and M. De Vittorio, "Stress-driven AlN cantilever-based flow sensor for fish lateral line system," Microelectronic Engineering, vol. 88, no. 8, pp. 2376-2378, 2011.

[13] V. I. Fernandez, S. M. Hou, F. S. Hover et al., "Lateral-lineinspired mems-array pressure sensing for passive underwater navigation," 2007.

[14] A. G. P. Kottapalli, M. Asadnia, J. M. Miao, G. Barbastathis, and M. S. Triantafyllou, "A flexible liquid crystal polymer MEMS pressure sensor array for fish-like underwater sensing," Smart Materials and Structures, vol. 21, no. 11, Article ID 115030, 2012.

[15] F. M. Yaul, V. Bulović, and J. H. Lang, "A flexible underwater pressure sensor array using a conductive elastomer strain gauge," Journal of Microelectromechanical Systems, vol. 21, no. 4, pp. 897-907, 2012. 
[16] M. Asadnia, A. G. P. Kottapalli, Z. Shen, J. Miao, and M. Triantafyllou, "Flexible and surface-mountable piezoelectric sensor arrays for underwater sensing in marine vehicles," IEEE Sensors Journal, vol. 13, no. 10, pp. 3918-3925, 2013.

[17] M. Asadnia, A. G. P. Kottapalli, J. Miao, M. E. Warkiani, and M. S. Triantafyllou, "Artificial fish skin of self-powered microelectromechanical systems hair cells for sensing hydrodynamic flow phenomena," Journal of the Royal Society Interface, vol. 12, no. 111, Article ID 20150322, 2015.

[18] A. T. Abdulsadda and X. Tan, "Underwater source localization using an ipmc-based artificial lateral line," in Proceedings of the IEEE International Conference on Robotics \& Automation (ICRA '11), vol. 19, no. 6, pp. 2719-2724, Shanghai, China, 2011.

[19] G. J. M. Krijnen, M. Dijkstra, J. J. van Baar et al., "MEMS based hair flow-sensors as model systems for acoustic perception studies," Nanotechnology, vol. 17, no. 4, pp. S84-S89, 2006.

[20] J. B. Stocking, W. C. Eberhardt, and Y. A. Shakhsheer, "A capacitance-based whisker-like artificial sensor for fluid motion sensing," in Proceedings of the IEEE Sensors, vol. 143, no. 2, pp. 2224-2229, Waikoloa, Hawaii, USA, November 2010.

[21] A. Klein and H. Bleckmann, "Determination of object position, vortex shedding frequency and flow velocity using artificial lateral line canals," Beilstein Journal of Nanotechnology, vol. 2, no. 1, pp. 276-283, 2011.

[22] H. Herzog, S. Steltenkamp, A. Klein, S. Tätzner, E. Schulze, and H. Bleckmann, "Micro-machined flow sensors mimicking lateral line canal neuromasts," Micromachines, vol. 6, no. 8, pp. 1189-1212, 2015.

[23] A. M. K. Dagamseh, T. S. J. Lammerink, M. L. Kolster, C. M. Bruinink, R. J. Wiegerink, and G. J. M. Krijnen, "Dipole-source localization using biomimetic flow-sensor arrays positioned as lateral-line system," Sensors \& Actuators A: Physical, vol. 162, no. 2, pp. 355-360, 2010.

[24] J. Chen, J. Engel, N. Chen, S. Pandya, S. Coombs, and C. Liu, "Artificial lateral line and hydrodynamic object tracking," in Proceedings of the 19th IEEE International Conference on Micro Electro Mechanical Systems (MEMS '06), pp. 694-697, Istanbul, Turkey, January 2006.

[25] P. Liu, R. Zhu, and R. Que, "A flexible flow sensor system and its characteristics for fluid mechanics measurements," Sensors, vol. 9, no. 12, pp. 9533-9543, 2009.

[26] S. Pandya, Y. Yang, C. Liu, and D. L. Jones, "Biomimetic imaging of flow phenomena," in Proceedings of the IEEE International Conference on Acoustics, Speech, Signal Processing (ICASSP '07), vol. 2, no. 2, pp. 933-936, Honolulu, Hawaii, USA, April 2007.

[27] L. DeVries, F. D. Lagor, H. Lei, X. Tan, and D. A. Paley, "Distributed flow estimation and closed-loop control of an underwater vehicle with a multi-modal artificial lateral line," Bioinspiration \& Biomimetics, vol. 10, no. 2, Article ID 025002, 2015.

[28] L. D. Chambers, O. Akanyeti, R. Venturelli et al., "A fish perspective: detecting flow features while moving using an artificial lateral line in steady and unsteady flow," Journal of the Royal Society Interface, vol. 11, no. 99, 2014.

[29] Y. Li, W. Wang, W. Luo et al., "Study on swimming modes sensing of robotic fish based on artificial lateral line system," Ordnance Industry Automation, vol. 34, no. 5, pp. 88-92, 2015 (Chinese).

[30] T. Salumäe and M. Kruusmaa, "Flow-relative control of an underwater robot," Proceedings of the Royal Society of London A: Mathematical, Physical and Engineering Sciences, vol. 469, no. 2153, Article ID 20120671, 2013.
[31] M. Kruusmaa, G. Toming, T. Salumäe et al., "Swimming speed control and on-board flow sensing of an artificial trout," in Proceedings of the IEEE International Conference on Robotics \& Automation (ICRA '11), vol. 19, no. 6, pp. 1791-1796, Shanghai, China, May 2011.

[32] Y. Yang, N. Chen, C. Tucker, and J. Engel, "From artificial hair cell sensor to artificial lateral line system: development and application," in Proceedings of the IEEE International Conference on Micro Electro Mechanical Systems (MEMS '07), pp. 577-580, Kobe, Japan, January 2007.

[33] Y. Yang, A. Klein, H. Bleckmann, and C. Liu, "Artificial lateral line canal for hydrodynamic detection," Applied Physics Letters, vol. 99, no. 2, Article ID 023701, 2011.

[34] F. M. Yaul, A Flexible Underwater Pressure Sensor Array for Artificial Lateral Line Applications, Massachusetts Institute of Technology, 2011.

[35] A. T. Abdulsadda and X. Tan, "Nonlinear estimation-based dipole source localization for artificial lateral line systems," Bioinspiration and Biomimetics, vol. 8, no. 2, Article ID 026005, 2013.

[36] T. Salumäe, I. Rano, O. Akanyeti, and M. Kruusmaa, "Against the flow: a Braitenberg controller for a fish robot," in Proceedings of the IEEE International Conference on Robotics and Automation (ICRA '12), pp. 4210-4215, St. Paul, Minn, USA, May 2012.

[37] G. H. Parker, "The function of the lateral-line organs in fishes," Bulletin of the U.S. Fish Commission, vol. 24, pp. 185-207, 1904.

[38] R. G. Northcutt, "The phylogenetic distribution and innervation of craniate mechanoreceptive lateral lines," in The Mechanosensory Lateral Line, pp. 17-78, Springer, New York, NY, USA, 1989.

[39] A. Flock and J. Wersall, "A study of the orientation of the sensory hairs of the receptor cells in the lateral line organ of fish, with special reference to the function of the receptors," The Journal of Cell Biology, vol. 15, pp. 19-27, 1962.

[40] S. Coombs, J. Janssen, and J. F. Webb, Diversity of Lateral Line Systems: Evolutionary and Functional Considerations. Sensory Biology of Aquatic Animals, Springer, New York, NY, USA, 1988.

[41] S. M. V. Netten, "Hydrodynamics of the excitation of the cupula in the fish canal lateral line," Veterinary Dermatology, vol. 18, no. 5, pp. 324-331, 1991.

[42] A. J. Kalmijn, Functional Evolution of Lateral Line and Inner Ear Sensory Systems, Springer, New York, NY, USA, 1989.

[43] S. M. Van Netten, "Hydrodynamic detection by cupulae in a lateral-line canal: functional relations between physics and physiology," Biological Cybernetics, vol. 94, no. 1, pp. 67-85, 2006.

[44] J. Engelmann, W. Hanke, and H. Bleckmann, "Lateral line reception in still- and running water," Journal of Comparative Physiology A: Neuroethology, Sensory, Neural, and Behavioral Physiology, vol. 188, no. 7, pp. 513-526, 2002.

[45] H. Bleckmann and R. Zelick, "Lateral line system of fish," Integrative Zoology, vol. 4, no. 1, pp. 13-25, 2009.

[46] S. R. Karumuri, K. G. Sravani, S. D. Sailaja, J. V. Sekhar, Y. Srinivas, and R. Bhattacharjee, "Micro-Electro-Mechanical-Systems (MEMS) technology," Archives of Applied Science Research, vol. 1, pp. 307-314, 2012.

[47] J. Zou, J. Chen, and C. Liu, "Plastic Deformation Magnetic Assembly (PDMA," in Transducers '01 Eurosensors XV: The 11th International Conference on Solid-State Sensors and Actuators June 10-14, 2001 Munich, Germany, pp. 1554-1557, Springer, Berlin, Germany, 2001. 
[48] F. Rizzi, A. Qualtieri, T. Dattoma, G. Epifani, and M. De Vittorio, "Biomimetics of underwater hair cell sensing," Microelectronic Engineering, vol. 132, pp. 90-97, 2015.

[49] S. Peleshanko, M. D. Julian, M. Ornatska et al., "Hydrogelencapsulated microfabricated haircells mimicking fish cupula neuromast," Advanced Materials, vol. 19, no. 19, pp. 2903-2909, 2007.

[50] A. T. Abdulsadda and X. Tan, "An artificial lateral line system using IPMC sensor arrays," International Journal of Smart and Nano Materials, vol. 3, no. 3, pp. 226-242, 2012.

[51] A. T. Abdulsadda, F. Zhang, and X. Tan, "Localization of source with unknown amplitude using IPMC sensor arrays," in Proceedings of the SPIE Smart Structures and Materials+ Nondestructive Evaluation and Health Monitoring, vol. 7976, no. 10, pp. 2378-2384, International Society for Optics and Photonics, Portland, Ore, USA, 2011.

[52] A. T. Abdulsadda and X. Tan, "Localization of a moving dipole source underwater using an artificial lateral line," in Proceedings of the Bioinspiration, Biomimetics, and Bioreplication, vol. 8839 of Proceedings of SPIE, San Diego, Calif, USA, March 2012.

[53] G. Krijnen, T. Lammerink, R. Wiegerink, and J. Casas, "Cricket inspired flow-sensor arrays," in Proceedings of the IEEE SENSORS Conference, pp. 539-546, Atlanta, Ga, USA, October 2007.

[54] A. Dagamseh, R. Wiegerink, T. Lammerink, and G. Krijnen, "Imaging dipole flow sources using an artificial lateral-line system made of biomimetic hair flow sensors," Journal of the Royal Society Interface, vol. 10, no. 83, pp. 82-86, 2013.

[55] Y. Yang, J. Chen, J. Engel et al., "Distant touch hydrodynamic imaging with an artificial lateral line," Proceedings of the National Academy of Sciences of the United States of America, vol. 103, no. 50, pp. 18891-18895, 2006.

[56] S. Pandya, Y. Yang, D. L. Jones et al., "Multisensor processing algorithms for underwater dipole localization and tracking using mems artificial lateral-line sensors," EURASIP Journal on Advances in Signal Processing, vol. 2006, Article ID 076593, 2006.

[57] B. P. Chagnaud, C. Brücker, M. H. Hofmann, and H. Bleckmann, "Measuring flow velocity and flow direction by spatial and temporal analysis of flow fluctuations," The Journal of Neuroscience, vol. 28, no. 17, pp. 4479-4487, 2008. 




\section{Enfincering}
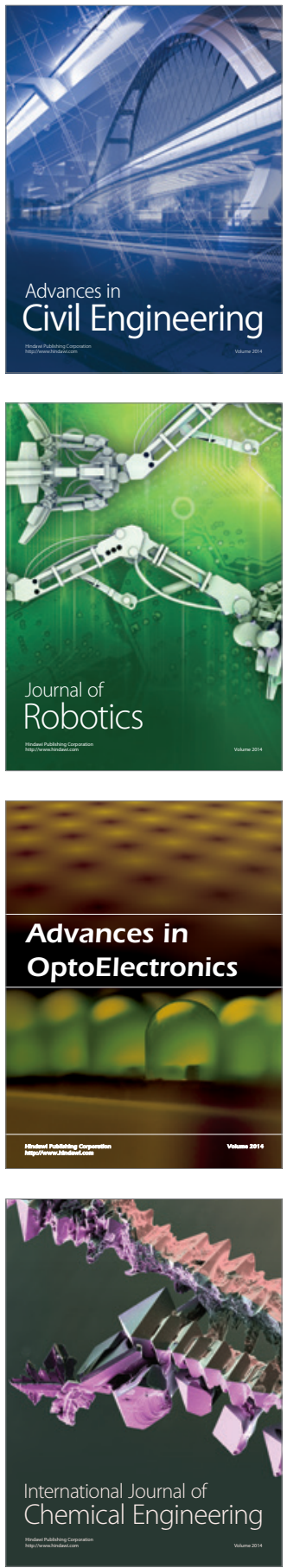

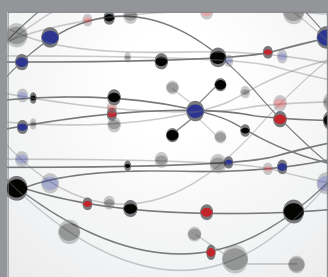

The Scientific World Journal

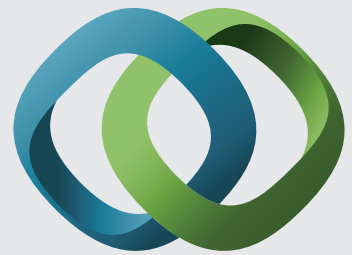

\section{Hindawi}

Submit your manuscripts at

http://www.hindawi.com
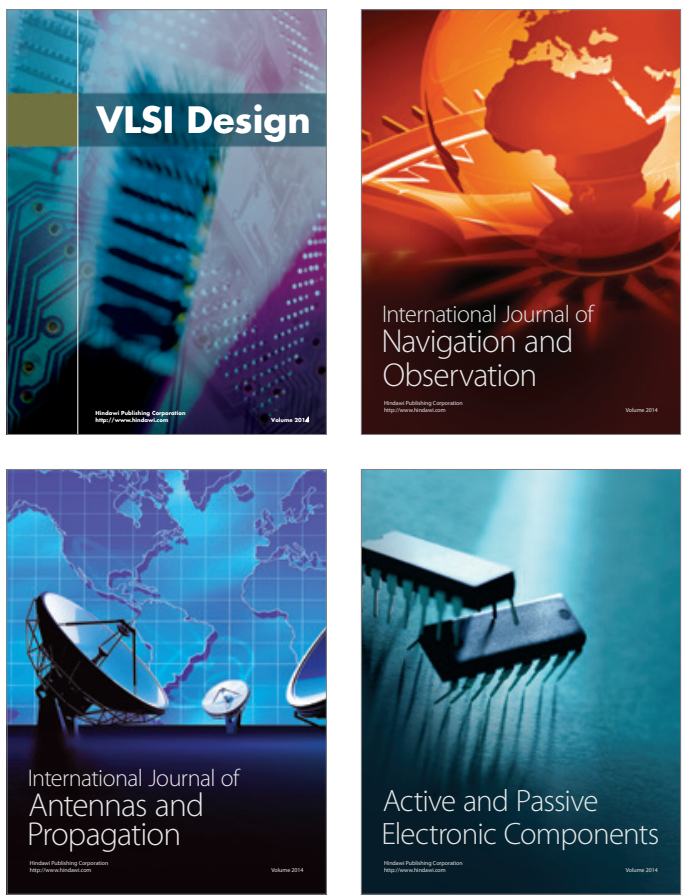
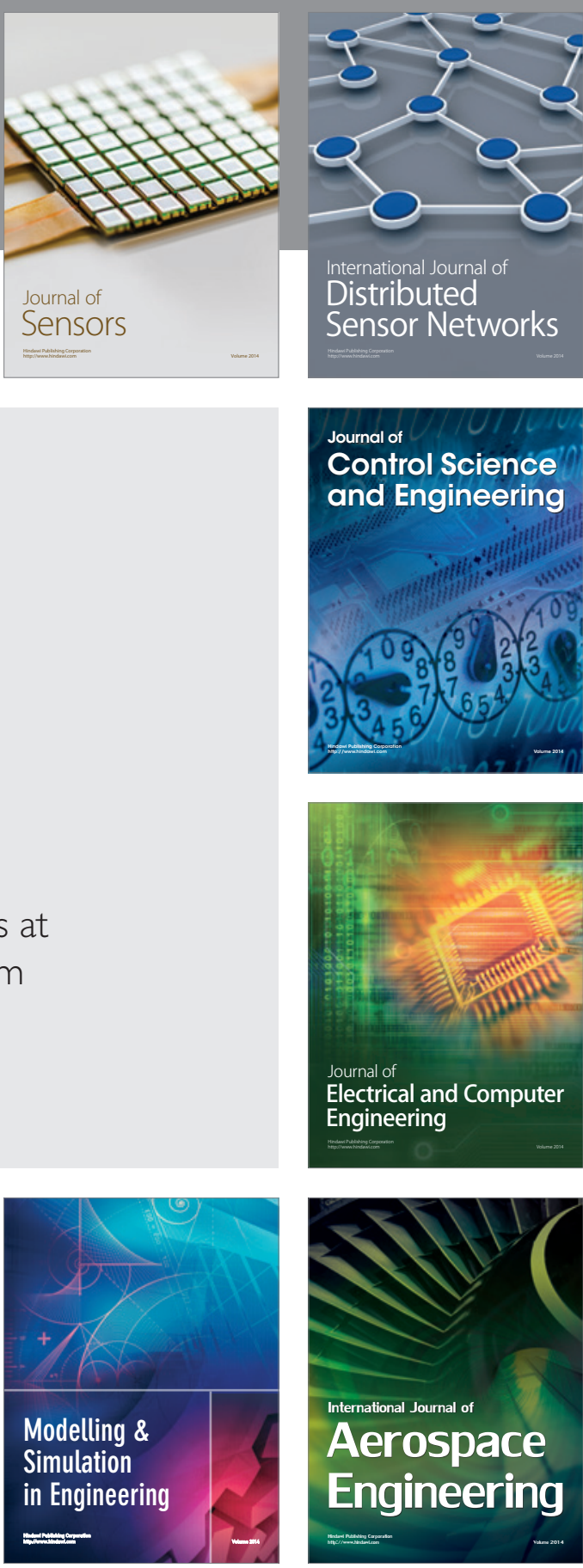

International Journal of

Distributed

Sensor Networks

Journal of

Control Science

and Engineering
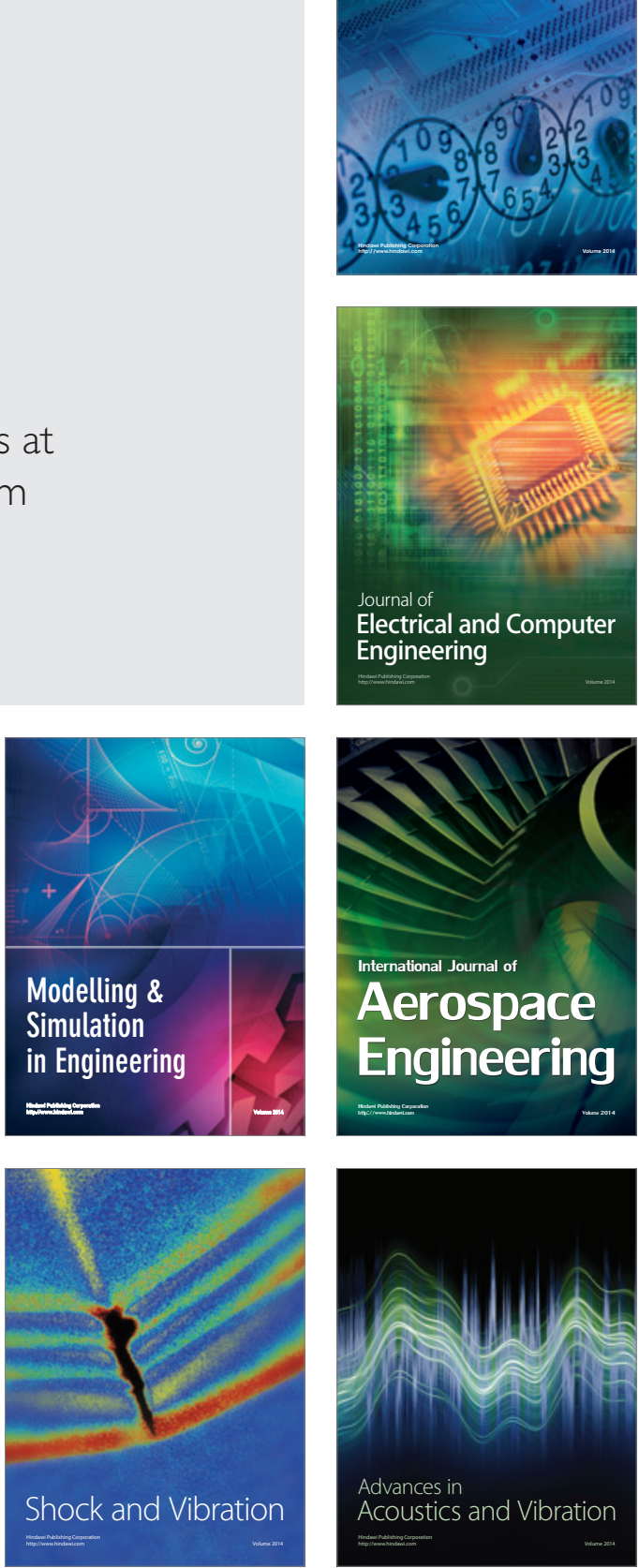